\title{
GOSS TEXTURE DEVELOPMENT IN Fe-Si STEELS
}

\author{
N. RAJMOHAN ${ }^{a}$, J.A. SZPUNAR ${ }^{a, *}$ and Y. HAYAKAWA ${ }^{b}$ \\ a Department of Mining and Metallurgical Engineering, McGill University, \\ 3610 University Street, Montreal, Quebec, Canada H3A 2B2; ${ }^{\text {b Technical }}$ \\ Research Laboratories, Kawasaki Steel Corporation, 1-Kawasakidouri, \\ Mizushima, Kurashiki, Okayama, Japan 712
}

(Received in final form 28 September 1997)

Goss texture development in silicon steels has been studied through EBSP measurements and various computer simulations and calculations. The results of these studies suggest the possible role of high energy grain boundaries (HEGB) in the abnormal growth of Goss grains. The Goss orientation has a fraction of HEGBs that is higher than any other commonly observed orientations in the primary recrystallized silicon steels. The HEGBs have high GB diffusion coefficients which cause rapid coarsening of precipitates on these HEGBs and release them earlier, at the time when other GBs are still pinned. A difference in the mobility between the HEGBs and the other GBs favours the abnormal growth of Goss grains. The Monte-Carlo methods that have been developed and used to validate this assumption have generated abnormally growing Goss grains. The experimentally observed grain boundary character distributions (GBCD) around the growing Goss grains have been reproduced in simulation by assuming high mobility to HEGBs. Apart from the high mobility differences between different GBs, the importance of the fraction of GBs with high mobility around growing Goss grains is realized.

Keywords: Goss texture; High energy grain boundaries; Precipitates; Monte-Carlo simulation

\section{INTRODUCTION}

Although the grain oriented silicon steels have been known since the work of Goss (1934), the mechanism of the development of Goss texture during secondary recrystallization (SR) is still under debate among researchers. Various mechanisms of the development of Goss texture

\footnotetext{
* Corresponding author.
} 
have already been proposed. One theory assumes that Goss grains form colonies in the case of conventional grain-oriented electrical steel in which the final cold rolling reduction is approximately $60 \%$. Coalescence of these colonies during annealing gives them the size advantage for further growth (Inokuti and Maeda, 1984; Matsuo, 1989). However, when GB energies and mobilities are uniform, it has been shown previously both by theoretical (Andersen et al., 1995a,b) and computational analyses (Anderson et al., 1984; Srolovitz et al., 1985; Hinz and Szpunar, 1995) that a very large grain will always grow more slowly than the average size grain and will eventually rejoin the normal size distribution. Therefore, the abnormal grain growth (AGG) can only occur when normal grain growth is inhibited. In conclusion, unless the abnormally growing grain enjoys some growth advantage other than size of its neighbours, SR will not be realized. In the case of high permeability steel, the Goss grains do not form colonies (Shimizu and Harase, 1989; Pease et al., 1985) and abnormal grain growth is observed in practice.

It is known that the grain growth in polycrystalline materials is entirely controlled by grain boundaries. The driving force for grain growth is the reduction of GB area, and thus the total energy of the system. The kinetics of grain growth are determined by grain boundary mobility. Among many factors such as surface energy, particle pinning, grain boundary grooving, etc., which may influence AGG, effect of second phase particles is considered to be of prime importance in the case of Goss texture development in $\mathrm{Fe}-\mathrm{Si}$ steels. At higher temperatures, precipitate particles undergo Oswald Ripening (OR). This causes the breakdown of GB pinning and favours certain GBs that move faster than others and are responsible for AGG.

Regarding the type of boundaries responsible for the growth of Goss grain in Fe-Si steel, one group of researchers (Harase et al., 1986; 1991; Shimizu et al., 1990; Abbruzzese et al., 1992; Lin et al., 1996) argue that certain coincidence site lattice (CSL) boundaries have high mobilities which result in AGG. Harase et al. (1991) and Shimizu et al. (1990) argued that among various CSL boundaries, $\Sigma 9$ played an important role in the development of $\left\{\begin{array}{llll}1 & 1 & 0\end{array}\right\}\left\langle\begin{array}{lll}0 & 0 & 1\end{array}\right\rangle$ texture. In their work a possible role of other CSL boundaries was not ruled out. Recently Lin et al. (1996) stated that the low $\Sigma$ boundaries including $\Sigma 3$ are responsible for the AGG of Goss grains in $\mathrm{Fe}-\mathrm{Si}$ steels. These authors argue that 
there is no energetic or structural basis for an enhanced stability of the $\Sigma 3$ boundary in b.c.c. materials such as $\mathrm{Fe}-\mathrm{Si}$. The fact is that the fraction of CSL boundaries other than the low angle grain boundary $(\Sigma 1)$ is very small, usually less than $10 \%$, (Harase and Shimizu, 1988; Shimizu and Harase, 1989) and it cannot be responsible for a major change in texture. Different type of CSL boundaries are given importance from case to case [ $\Sigma 9$ (Harase et al., 1991), $\Sigma 7$ (Harase, 1992), $\Sigma 5$ (Yoshitomi et al., 1993)]; however, no explanation for the selection mechanism has been offered. Although a lot of work has been published on the possible role of CSL boundaries on AGG, this has not been confirmed satisfactorily or accurately.

Dependence of GB energy on the GB misorientation was recognized a long time ago. Dunn and Lionetti (1949) and Dunn et al. (1950a,b) measured the GB energy as a function of misorientation angle for $\langle 100\rangle$ and $\left\langle\begin{array}{llll}1 & 1 & 0\end{array}\right\rangle$ tilt boundaries in $\mathrm{Fe}-\mathrm{Si}$ steels. The measured relative GB energy is shown in Fig. 1. The graph shows that the GBs with middle misorientation namely $20^{\circ}-45^{\circ}$ are HEGBs. The GBs with misorientations in other ranges namely $<20^{\circ}$ and $>45^{\circ}$ possess low energies. The direction of GB migration is dictated by the GB energies. However, the thermal activation energy of GB migration controls the kinetics of grain growth. It is observed that the dependence of the activation energy on GB misorientation is different at various concentrations of impurities. The results of Aust and Rutter (1959a,b) indicate that in high purity metals, the differences in GB mobility arise primarily from an orientation dependence of solute segregation to the boundary, rather than an intrinsic difference between GB mobilities. These authors demonstrated that the CSL boundaries are more mobile than random boundaries up to an impurity level of $0.006 \mathrm{wt} \% \mathrm{Sn}$ in $\mathrm{Pb}$. At higher impurity levels the difference between mobilities of CSL and random boundaries disappear (Humphreys and Hatherly, 1995). Again, it has been reported that the speciality of the CSL boundaries vanishes at higher temperatures (Humphreys and Hatherly, 1995). As a typical $\mathrm{Fe}-\mathrm{Si}$ steel has around 3\% $\mathrm{Si}$ with other alloying elements such as $\mathrm{C}$, $\mathrm{Mn}, \mathrm{S}, \mathrm{Al}, \mathrm{N}$, etc., in considerable amount and the secondary recrystallization process is observed to happen above $1000^{\circ} \mathrm{C}$, the special property of CSL boundaries is not necessarily preserved for this case.

In an effort to prove the underlying mechanism of the Goss texture development in $\mathrm{Fe}-\mathrm{Si}$ steel, various textural and microstructural 


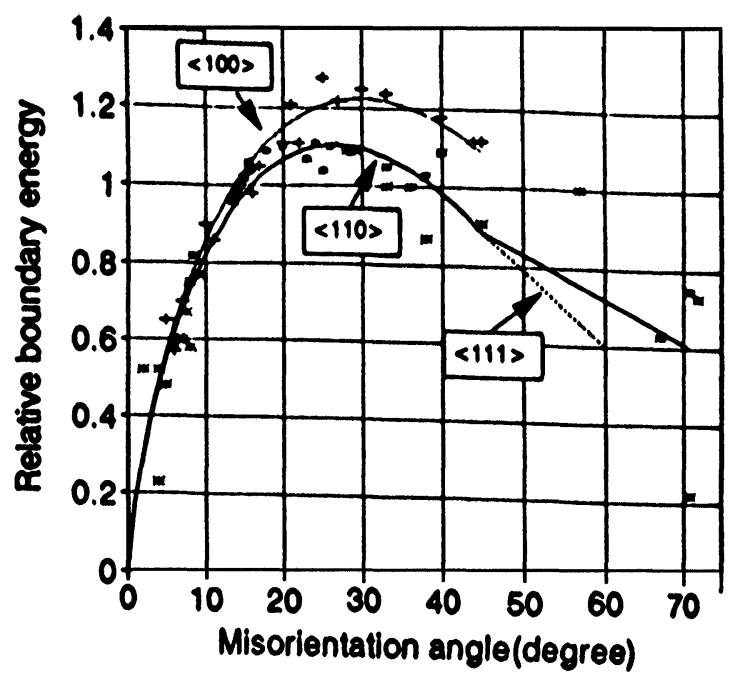

FIGURE 1 The grain boundary energy vs. misorientation angle for $\langle 100\rangle,\langle 110\rangle$ and $\langle 111\rangle$ tilt boundaries in $\mathrm{Fe}-3 \% \mathrm{Si}$ steel.

aspects of grain growth have been studied by our group (Hayakawa and Szpunar, 1997a,b; Hayakawa et al., 1996; 1997; Rajmohan et al., 1997). These studies using texture measurements, OIM and computer simulation methods reveal the importance of HEGBs. The purpose of this article is to explain the possible role of HEGB in the development of Goss texture in silicon steels. Previously, an experimental study on commercial purity single crystalline specimens of $\mathrm{Fe}-3 \% \mathrm{Si}$, using orientation measurements and grain misorientation analyses of pole figures conducted by Titorov $(1973 ; 1994)$ also concluded that GBs with middle misorientation angles were responsible for AGG of the Goss grain. Also, the experimental findings by Watanabe (1992) demonstrate that the large growing grains have a high number of HEGBs. At high concentrations of alloying elements and at high processing temperatures, the HEGBs have the highest mobility and the highest precipitate coarsening rate and consequently are released for migration earlier than other boundaries in an alloy system. The high mobility of the HEGB and low mobility of the low energy grain boundaries (LEGB) in industrial purity metals is observed even if the precipitates are not present and can be explained using vacancy mechanisms. It is well known that the GB migration takes place 
because of the exchange of atoms between grains through vacancies (Gottstein and Schwarzer, 1992; Ralph et al., 1992; Babcock and Balluffi, 1989). The HEGB is more capable of absorbing and emitting vacancies (Hahn and Gleiter, 1980) because the number of vacancies is related to the disorder in the grain boundary structure. The HEGB has a more disordered structure, therefore a high number of vacancies and dislocations should contribute to a high mobility at high impurity levels and at high processing temperatures. The following sections of this article delineate our investigations made to reveal the mechanism of Goss texture development in both conventional and high permeability $\mathrm{Fe}-\mathrm{Si}$ steels.

\section{THE ROLE OF THE HIGH ENERGY GRAIN BOUNDARY}

An investigation made by Hayakawa et al. (Hayakawa et al., 1996; Hayakawa and Szpunar, 1997a) through GBCD analyses on both conventional and high permeability steels after primary recrystallization paved the way for identifying the role of HEGBs on the development of Goss texture in those materials. The calculated orientation distribution function (ODF) from the measured pole figures (110), (200) and (211) for both these steels are shown in Fig. 2. To calculate the GBCD, pairs of grains have been generated in proportion to the value of the ODF using the method proposed by Morawiec et al. (1993). To investigate grain boundaries around the grain having a certain specific orientation, the orientation of the selected grain is fixed, and the orientation of the neighbouring grain was generated using the ODF data. The calculated GBCD using $10^{5}$ pairs of grains has an error less than $1 \%$. Misorientation angles, which are the minimum angle among the equivalent 24 angles and rotation axis, have been calculated using the unit quaternion (Grimmer, 1974). The validity of the statistical calculation of GBCD has been tested previously (Hayakawa et al., 1996), by comparing the result obtained with direct measurement of misorientation of grains using the EBSP. The portion of CSL boundaries obtained from the measurement of 511 grains in the high permeability steel specimen shows good agreement with the calculation except for the $\Sigma 1$ boundary. The percentage of the $\Sigma 1$ boundary is higher than the calculated value, and the main reason for such a 


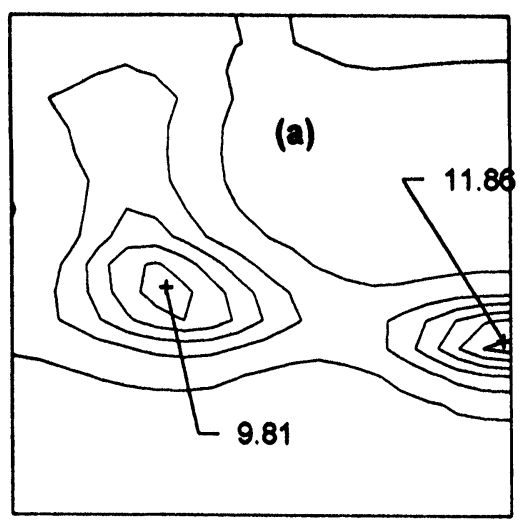

Contour Levels: $1.0 \quad 3.0 \quad 5.0 \quad 7.0 \quad 9.0 \quad 11.0$

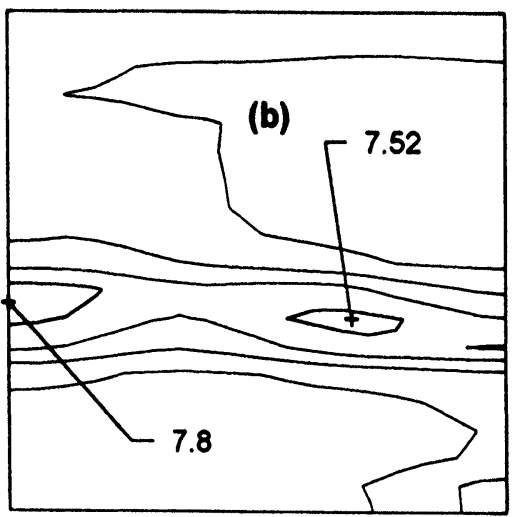

Contour Levels: $1.0 \quad 3.0 \quad 5.07 .0$

FIGURE 2 The ODF of (a) high permeability steel and (b) conventional electrical steel.

difference is that $\Sigma 1$ boundaries tend to form colonies that cannot be predicted from the ODF analysis.

Suppose that the fraction of boundaries having misorientation angle $\omega$ lying between certain angles $A$ and $B$ for a grain having a specific orientation $g$ is defined by the following expression:

$$
\text { Fraction }(A<\omega<B, g)=\{N(A<\omega<B, g) / N(g)\},
$$


where $N(A<\omega<B, g)$ is the number of boundaries that have misorientation angle $(A<\omega<B)$ around the grain having a specific orientation $g$, and $N(g)$ is the total number of generated boundaries around the grain having the same specific orientation $g$. The fraction of grains having misorientation angle $\omega$ with respect to the Goss grain, the grain having average orientation, and the grain representing the main texture component are shown in Fig. 3. The main texture components after primary recrystallization are $\left\{\begin{array}{lll}1 & 1 & 1\end{array}\right\}\langle 112\rangle$ and $\left\{\begin{array}{lll}1 & 1 & 1\end{array}\right\}\langle 110\rangle$ for the high permeability steel and conventional steels respectively. The main difference between distributions illustrated in Fig. 3 is that the Goss grain has more intermediate misorientation angle $\left(20^{\circ}<\omega<45^{\circ}\right)$ grain boundaries and less low angle $\left(15^{\circ}>\omega\right)$ and high angle $\left(45^{\circ}<\omega\right)$ grain boundaries than a grain having an average orientation or the grain of the main texture component. Figure 3 also shows that grains with orientation of the main texture component have fewer intermediate misorientation angle GBs and more low and high angle GBs than the grain representing the average texture. This observation is true for both the conventional and high permeability steels. The fraction of middle misorientation $\left(20^{\circ}<\omega<45^{\circ}\right)$ grain boundaries around grains of different orientations can be represented in the Euler angle space and is shown in Fig. 4. For the high permeability steel, the fraction shows a sharp maximum which is $76 \%$ of the Goss orientation and a minimum which is $37 \%$ of the main texture component. A spectacular difference of $\sim 40 \%$ is seen between Goss orientation and main texture component. In the case of conventional electrical steel, though the Goss orientation has a higher fraction $(\sim 68 \%)$, the peak value of $70 \%$ fraction is found at the orientation which is $20^{\circ}$ deviated from Goss orientation through the $N D \|\langle 110\rangle$ axis.

The fraction of CSL boundaries around a grain having specific orientation $g$ can be estimated from EBSP measurement. According to the recent measurement by Lin et al. (1996), the fraction of $\Sigma 3-\Sigma 9 \mathrm{CSL}$ boundaries around Goss orientation after primary recrystallization is $\sim 13 \%$. The next highest fraction of $\Sigma 3-\Sigma 9$ CSL boundaries reported is $\sim 10 \%$ for $\left\{\begin{array}{llll}1 & 1 & 1\end{array}\right\}\left\langle\begin{array}{lll}1 & 1 & 2\end{array}\right\rangle$ in conventional electrical steel. These two orientations have a difference in the fractions of these boundaries only $3 \%$. From this data, it is also evident that if $\Sigma 3-\Sigma 29$ CSL boundaries are considered then the Goss and $\left\{\begin{array}{llll}1 & 1 & 1\end{array}\right\}\left\langle\begin{array}{lll}1 & 1 & 2\end{array}\right\rangle$ orientations have the fraction of $20 \%$ and $19 \%$ respectively. These values are close enough to 

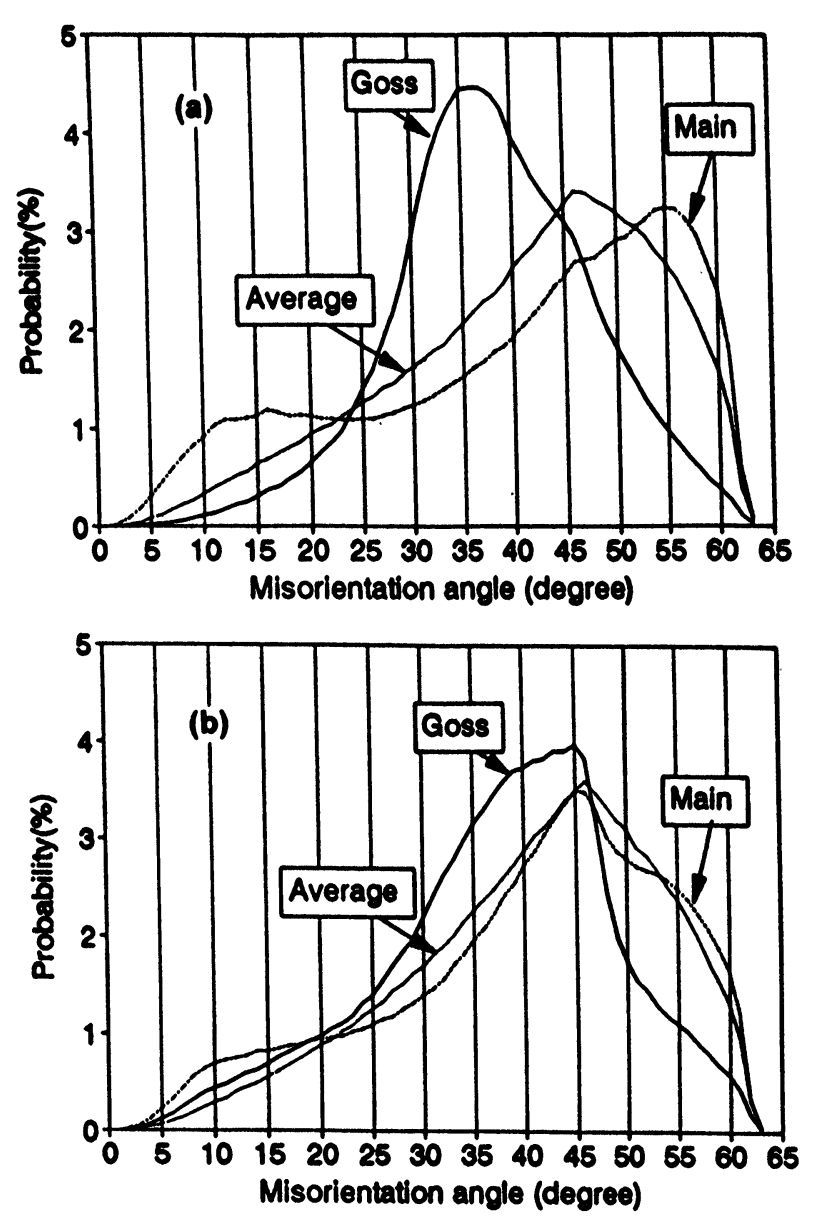

FIGURE 3 The misorientation angle distribution around the Goss, main and average texture components in (a) high permeability electrical steel (b) conventional electrical steel.

argue against the influence of CSL boundaries on the Goss texture development in $\mathrm{Fe}-\mathrm{Si}$ steels. From the computer calculations described above, made by Hinz and Szpunar (1995) and Hayakawa and Szpunar (1997a) a similar conclusion was reached. The fraction of CSL boundaries around the Goss grains is higher than the grain having average orientation, however, grains having other orientations such as $\left\{\begin{array}{lll}1 & 1 & 1\end{array}\right\}\left\langle\begin{array}{lll}0 & 1 & 1\end{array}\right\rangle$ and $\left\{\begin{array}{llll}2 & 1 & 0\end{array}\right\}\left\langle\begin{array}{lll}0 & 0 & 1\end{array}\right\rangle$ have higher or similar fractions of CSL. 
(a)

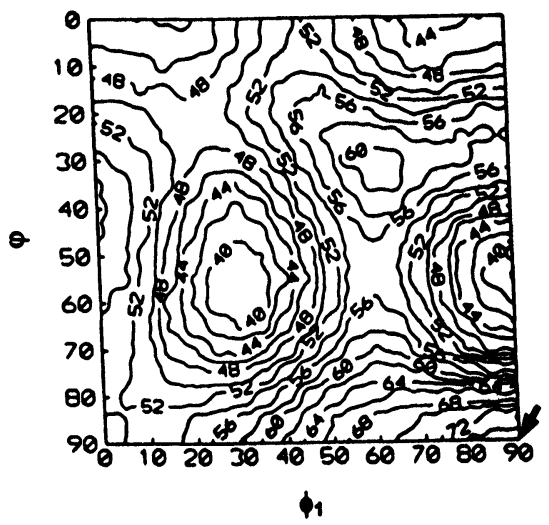

(b)

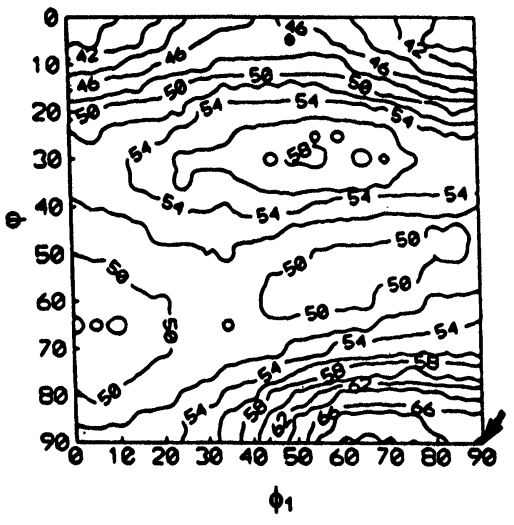

FIGURE 4 The fraction (\%) of GBs with misorientation angle between $20^{\circ}$ and $45^{\circ}$ around grains having various orientation represented in Euler angle space for $\phi_{2}=45^{\circ}$ section. (a) High permeability electrical steel and (b) conventional electrical steel.

The most important observation of their finding is that the absolute difference in fraction is at best only $3 \%$. The difference is too small to make any significant change in the secondary recrystallization mechanism. As far as an individual CSL boundary such as $\Sigma 9$ is concerned, the number of such boundaries surrounding the Goss grain is approximately $2 \%$, which is too small to alter the primary recrystallized texture and influence the development of Goss texture. On the other hand the difference in the middle misorientation boundaries is as high as $40 \%$ between the Goss and main orientations in both the conventional and high permeability steels.

\section{COMPUTER SIMULATION OF GOSS TEXTURE DEVELOPMENT}

The development of the secondary recrystallization texture is controlled by the precipitates and without them, AGG of Goss grains does not happen. Interaction between the precipitates and the grain boundaries, therefore, is more important than the inherent mobility 
differences between different grain boundaries. Secondary recrystallization is often related to the coarsening and depletion of precipitates, which is a diffusion controlled process. As the GB diffusion rate is higher than the bulk diffusion rate the coarsening of precipitates starts at the GB. Attempts have been made (Borisov et al., 1964) to relate the GB energy and GB diffusion. The theoretical approach developed by Borisov et al. (1964) has been verified on various materials (Pelleg, 1966) and found to correlate well with the experiments. The GB diffusion rate is a function of the GB misorientation angle, which is similar to the GB energy. It also reported that coarse precipitates were frequently found inside large growing Goss grains than within small grains (Hayakawa and Szpunar, 1997a). This may explain the fact that precipitates grow faster at the boundary of the growing grain. It is expected that HEGB has a high diffusion coefficient, leading to the acceleration of Oswald ripening of the precipitates.

Based on the observation described above, a model of anomalous grain growth has been proposed by Hayakawa and Szpunar (1997a). It assumes that, at the beginning of annealing, all the grain boundaries are pinned by precipitates. The rate of coarsening of precipitates is higher for HEGBs. Therefore, at the early stages of annealing, only the HEGB moves when the precipitates on this boundary coarsen to some critical size for unpinning. Based on this assumption, only the boundaries having a higher energy than a certain critical value $\left(E_{\mathrm{c}}\right)$ can move. At the early stage of the annealing, when the value of $E_{\mathrm{c}}$ is high, the growth advantage is small, thus we can expect normal grain growth, which is usually observed before the onset of secondary recrystallization. As the annealing progresses, the coarsening of precipitates continues such that the precipitates which are situated on a LEGB also reach the critical size for unpinning and such a GB begins to move. Therefore, it is logical to assume that during annealing, the value of $E_{\mathrm{c}}$ decreases with time.

This model has been incorporated in a Monte-Carlo simulation procedure (Hayakawa and Szpunar, 1997b) and the grain growth process has been monitored. The computer specimen consists of 1500 voronoi polyhedra grains modelled using half a million sites of a three dimensional honeycomb lattice. The grain orientations in this computer specimen represents the primary recrystallization texture of high permeability grain-oriented electrical steels. Each grain orientation is 
generated by the method proposed by Morawiec et al. (1993) in proportion to the value of ODF. The computer program incorporates both GB energy and GB mobility. The GB mobility is assumed to depend on GB energy $E$ as follows:

$$
\left.\begin{array}{l}
\text { mobility }=1, \text { when } E>E_{\mathrm{c}} \\
\text { mobility }=0, \text { when } E<E_{\mathrm{c}}
\end{array}\right\}
$$

where $E_{\mathrm{c}}$ is the critical value of boundary energy, and only the boundaries having energy higher than $E_{\mathrm{c}}$ can move. The decrease of $E_{\mathrm{c}}$ as the annealing proceeds is given by the following equation:

$$
E_{\mathrm{c}}=1.2-M C S \times 10^{-4} / a
$$

where, 1.2 represents the value of the highest relative boundary energy of the $\mathrm{Fe}-\mathrm{Si}$ system, $M C S$ is the number of Monte-Carlo steps and $a$ is a constant which is related to the rate of coarsening of precipitates and the values of 1, 2, 3, 4,6,8 are used. At high values of $a, E_{\mathrm{c}}$ decreases slowly during simulation. Expression (3) assumes that all the boundaries are pinned at the beginning of the simulation. As the annealing proceeds, the HEGBs are released at the early stages and at the latter stages even the LEGBs start to move. From the initial distribution of 1500 grains in this computer specimen, the average relative energy of all the grains is 0.97 . Goss grains are surrounded by HEGBs and the average boundary energy is 1.03 , while the main texture component grains are surrounded by higher number of LEGB with an average boundary energy of 0.92 . The relative volume of the largest grain with respect to average grain volume for various simulations with different $a$ values as expressed above are shown in Fig. 5. The near Goss grain dominates the whole area of a half million sites used in this MonteCarlo simulation except in two cases where the rate of decrease of $E_{\mathrm{c}}$ is the highest $(a=8)$ or the lowest $(a=1)$. Hayakawa and Szpunar (1997b) have also found that by using these procedures the Goss grain is selected among the 1500 grains for the AGG. The faster the decrease of $E_{\mathrm{c}}$, the shorter the incubation time for abnormal grain growth. When the rate of decrease of $E_{\mathrm{c}}$ is the highest, the grain growth behaviour observed in this simulation is close to the normal grain growth. This 


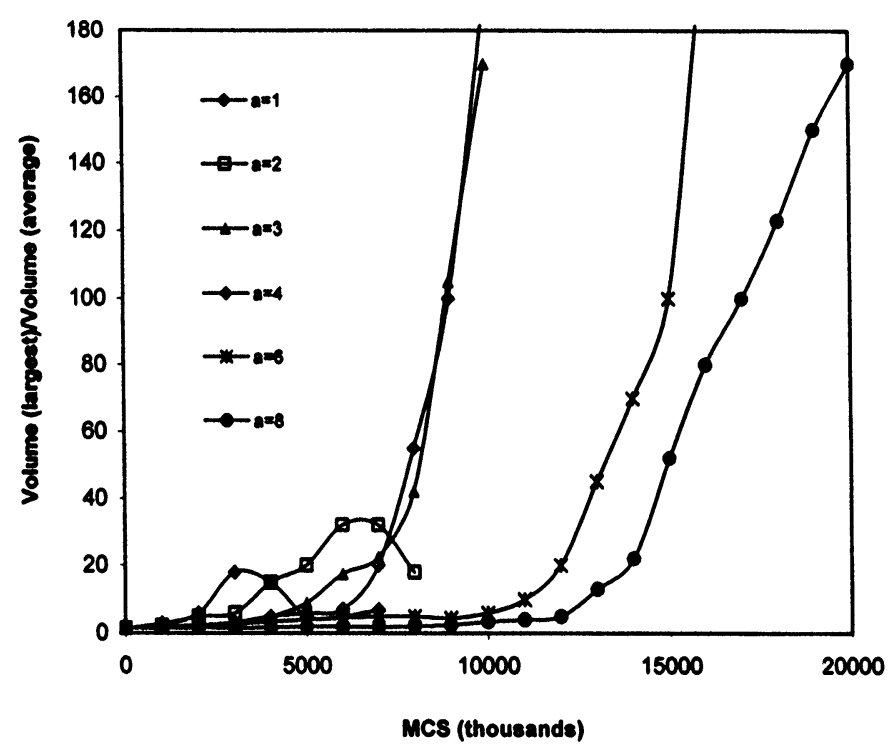

FIGURE 5 Relative volume of the growing largest grain for various $a$ 's in the Monte-Carlo simulation by Hayakawa and Szpunar (1997b).

corresponds to the failure of secondary recrystallization that results from the lack of necessary inhibition by coarse precipitates at the early stages of annealing. The change of ODF intensity values $f(g)$ of the main texture component $\left\{\begin{array}{llll}1 & 1 & 1\end{array}\right\}\left\langle\begin{array}{lll}1 & 1 & 2\end{array}\right\rangle$, and that of the Goss orientation are shown in Fig. 6 for both experiment and simulation. The ODF of the simulated texture is calculated from individual orientation of grains using Gaussian distribution (Bunge, 1982). Both results show an initial increase of the main texture component and simultaneous decrease in the strength of Goss texture.

Previous to this work the effect of CSL boundaries on the development of Goss texture in SR was studied using the three dimensional Monte-Carlo model with a full description of the microstructure and the crystallographic texture which was developed by Hinz and Szpunar (1995). The conclusion from that work is that the Goss texture could not develop under the condition that the CSL boundaries have high mobilities and low energies. Also, a role of the initial grain size advantage for the Goss grains has been studied. The assumption of high mobility to CSL boundaries and grain size advantage at the 

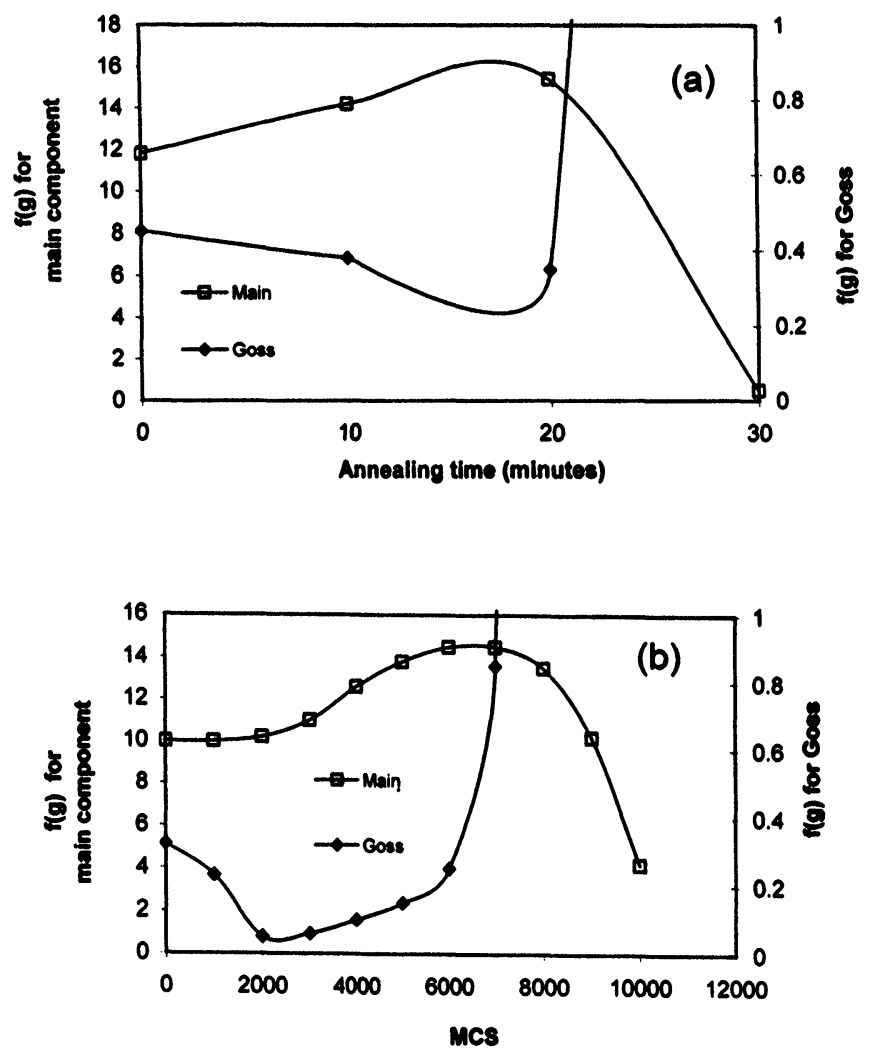

FIGURE 6 Computer simulation and experimentally measured texture intensities of Goss and main texture components during annealing (Hayakawa and Szpunar, 1997b).

beginning of the process, were not sufficient to simulate the anomalous growth of Goss grains in grain-oriented electrical steel.

\section{IMPORTANCE OF MOBILE BOUNDARY FRACTIONS}

Grain growth during annealing of polycrystalline materials is influenced by the type of GBs the grain encounters as it grows. Frequently, it has been noticed in the Monte-Carlo simulation of AGG that Goss grains that are smaller than other available grains in the computer specimen start growing abnormally. This elucidates the fact that not 
only the size of the nucleus grain is important but also the type and number of mobile GBs surrounding that grain. In a recent work, Rajmohan et al. (1997) explained the importance of fractions of mobile boundaries around the growing Goss grain for the AGG in Fe-Si steels using simple computer experiments.

In the present work, the growth of the Goss grain in the primary recrystallized matrix is followed under two different mobility assumptions chosen to verify both the 'CSL' and the 'middle misorientation boundary' theories. At the start of simulation, the primary recrystallized grain structure is assumed to be an array of tetrakaidecahedra. Each tetrakaidecahedron grain having the size of a lattice unit of the computer specimen has 14 neighbours (Smith, 1952). The relative mobility of grain boundaries is assumed to be very high for a particular type of GB, either CSL or middle misorientation GBs depending on the assumption that is made to study the texture development. Other boundaries, or so called 'forbidden' boundaries have a very low mobility. Based on the assumed mobility conditions, if a neighbouring grain has an 'allowed' misorientation with the growing grain, then the orientation of the neighbour becomes the same as the growing grain. If the misorientation is 'forbidden', the growth of the grain is stopped along that direction. At any particular time, GBCD of the growing grain can be computed based on orientations of its neighbours. One should note that if an active grain grows approximately in a spherical fashion, then the total number of tetrakaidecahedra (Smith, 1952) neighbours at each step of its growth is dictated by the following expression:

$$
12 n^{2}+2 \text { where } n=1,2,3, \ldots
$$

The above expression assumes that at each step, only the nearest neighbours are consumed. Each lattice unit is shared by 14 other lattice units, and therefore in order to avoid recounting of such units, the average effective number of neighbours per lattice unit consumed by the growing grain is approximated by an integer $N(1<N \leq 3.57) . N$ is the ratio between the total number of nearest neighbours of the growing grain, and the current peripheral lattice units covering the growing grains. Various experimental observations show that a grain growing in an abnormal fashion seldom grows spherically, but rather 
has a complex shape which is modified at every step during the course of its growth due to the anisotropic GB mobilities. This justifies the assumption that during the abnormal grain growth the unit tetrakaidecahedra grain maintains approximately the same average number of neighbours. The simple computer procedure employed in this work simulates the growth conditions during the initial stages of the AGG for both CSL and middle misorientation GB theories by assuming a different average number of non-interfering neighbours $N=2$ or 3 . As mentioned earlier, the average number of non-interfering neighbours can be defined as an integer which denotes the assumed number of nearest neighbours per lattice unit consumed by the growing grain. The kinetics of the growing grain in the computer experiment can be expressed by recording the volume of the grain as a function of growth step. The volume of a grain is the number of lattice units it has. In each growth step, all the peripheral lattice units which bound the growing grain are allowed to grow further depending on the type of boundary.

Based on this methodology, several computer experiments are carried out using an assumption that the HEGBs with $20^{\circ}-45^{\circ}$ grain misorientation are assumed to be of the type 'allowed' and others belong to the type 'forbidden'. The conclusion is that the Goss orientation grows considerably faster than other orientations in both the high permeability and conventional steels. These computer experiments made for an average of 3 non-interfering neighbours per lattice unit gives the GBCD around the abnormally growing Goss grain. A comparison of GBCD around growing Goss grains obtained by experimental and computation methods illustrated in Figs. 7 and 8 show a reasonable agreement for both conventional and high permeability steels, respectively. The experimental GBCDs are taken from Lin et al. (1996) and Hayakawa et al. (1997) for the conventional and high permeability steels, respectively.

It is also necessary to point out that the similar computer experiments carried out using the assumption that CSL boundaries from $\Sigma 3-$ $\Sigma 51$ ( $\Sigma 3$ is also included after Lin et al. (1996)) are 'mobile', show thàt Goss grains do not grow. Assuming two or three non-interfering neighbours per lattice unit, none of the existing grain orientations are found to grow abnormally. At the end of the grain growth, all the CSL boundaries are consumed and the specimen is left only with boundaries other than CSL which are considered to be of the 'forbidden' type. 


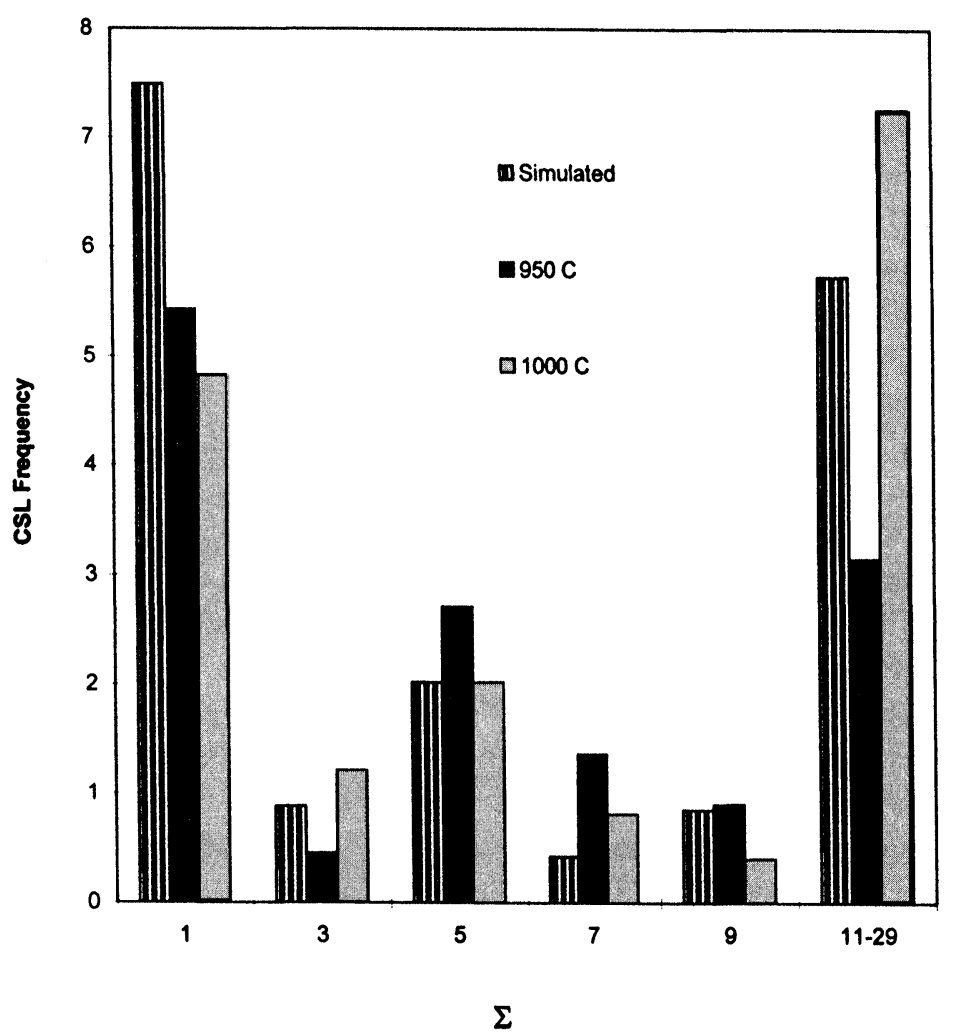

FIGURE 7 Experimentally determined GBCD bounding growing Goss grains and the simulated GBCD for the conventional $\mathrm{Fe}-3 \% \mathrm{Si}$ steel.

Therefore, the frequencies of all the CSL boundaries diminish to zero at this stage. A comparison between the GBCD obtained in this computer experiment with that observed experimentally by Lin et al. (1996) can be used to argue against the 'CSL boundary' theory.

It is important to point out that Rajmohan et al. (1997) have emphasized the concept of two kinds of fractions of mobile boundaries that are important for the grain growth. The first is called the matrix fraction (MF) of mobile boundaries for a given grain orientation and is defined as the average initial fraction of mobile boundaries the given grain has with other grains having orientations defined by the ODF. The MF is a constant for a given orientation of grain and given texture. 


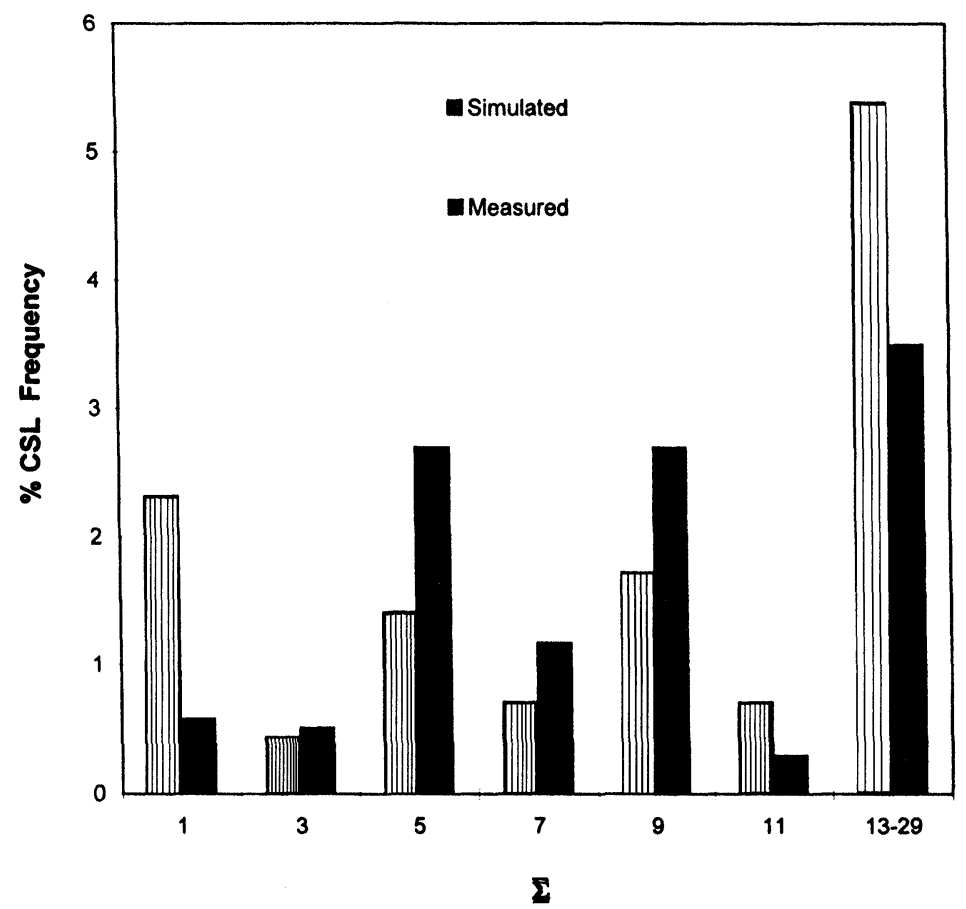

FIGURE 8 Experimentally determined GBCD bounding growing Goss grains and the simulated GBCD for the high permeability $\mathrm{Fe}-3 \% \mathrm{Si}$ steel.

As an example, the MF of Goss orientation in the matrix of primary recrystallized high permeability steel, under the assumption that middle misorientation angle boundaries are 'mobile', is about $76 \%$. One can introduce now the peripheral fraction (PF), which can be defined as the average fraction of mobile boundaries bounding a growing grain at any instant of the growth process. Contrary to MF, the PF varies as the grain grows and reaches a steady state after a certain number of growth steps, provided that the simultaneous change of texture in the matrix during annealing is not significant.

According to this analysis, the anomalous grain growth requires a high MF. For a given temperature, the higher the MF, the higher is the growth rate. As far as PF is concerned, it reaches a steady state after initial fluctuations. This steady state PF value is always smaller than the MF because as a grain grows the 'forbidden' boundaries are always 
attached to its periphery and the effective fraction of 'allowed' boundaries at the periphery diminishes. This fact is demonstrated by monitoring the growth of a given grain in a matrix of various MF. Figs. 9 and 10 show the growth behaviour of the growing grain for different fractions of 'allowed' boundaries and for the average number

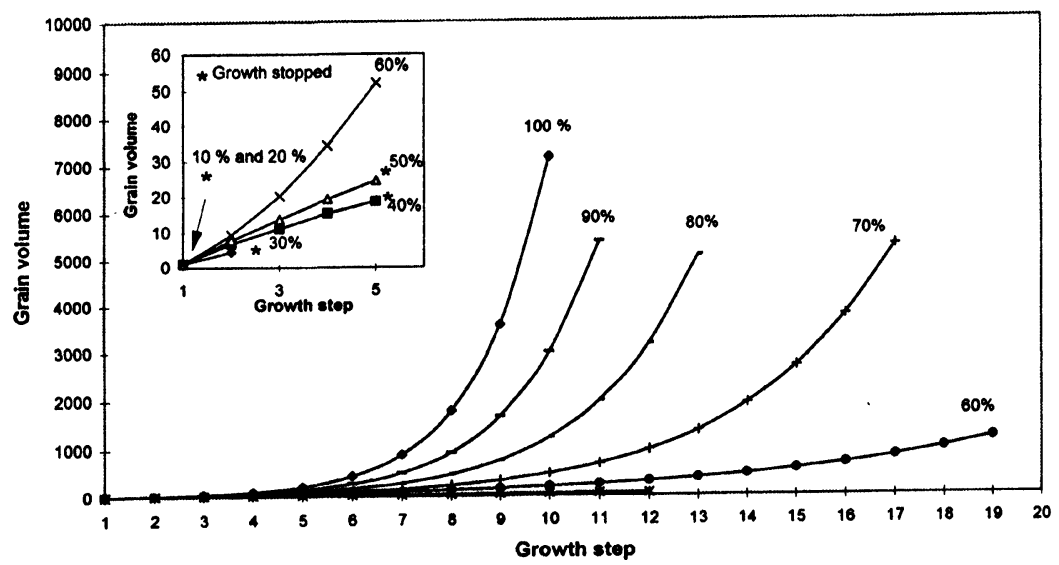

FIGURE 9 Growth behaviour for average number of non-interfering neighbours of 2 for different matrix fractions (\%) of 'allowed' boundaries. The inset shows the growth behaviour for the lower matrix fractions.

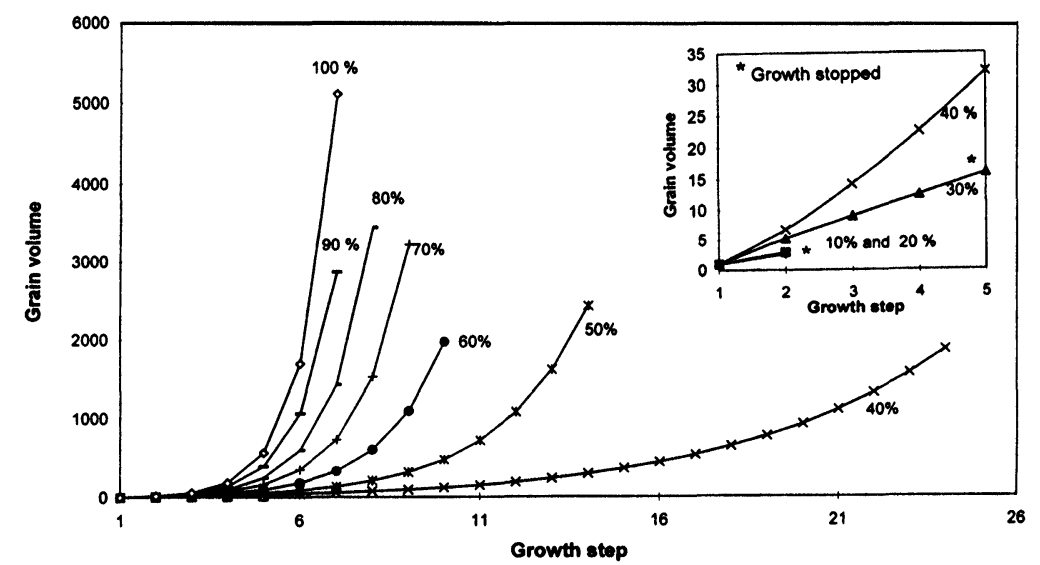

FIGURE 10 Growth behaviour for the average number of non-interfering neighbours of 3 for different matrix fractions (\%) of 'allowed' boundaries. The inset shows the growth behaviour for the lower matrix fractions. 
of non-interfering neighbours of 2 and 3, respectively. In both cases, each growth curve is obtained for a constant MF of the boundaries throughout the computation. Under such assumptions for the case of 2 non-interfering neighbours, the critical MF of mobile boundaries is close to $60 \%$. In the case of 3 non-interfering neighbours, the critical MF of mobile boundaries is around $40 \%$. The orientation which has a MF value lower than the critical value would ultimately stop growing after reaching the PF value of zero. Based on a computer analysis of grain misorientation, Hayakawa et al. (1996) and Hayakawa and Szpunar (1997a) pointed out that the MF for the Goss orientation is about $68 \%$ and $76 \%$ in the primary recrystallized matrix of the conventional and the high permeability electrical steels, respectively. In the case of conventional electrical steel, the average steady state PF for 10 different computer experiments with an average number of noninterfering neighbours of 2 is shown in Fig. 11. Under such conditions,

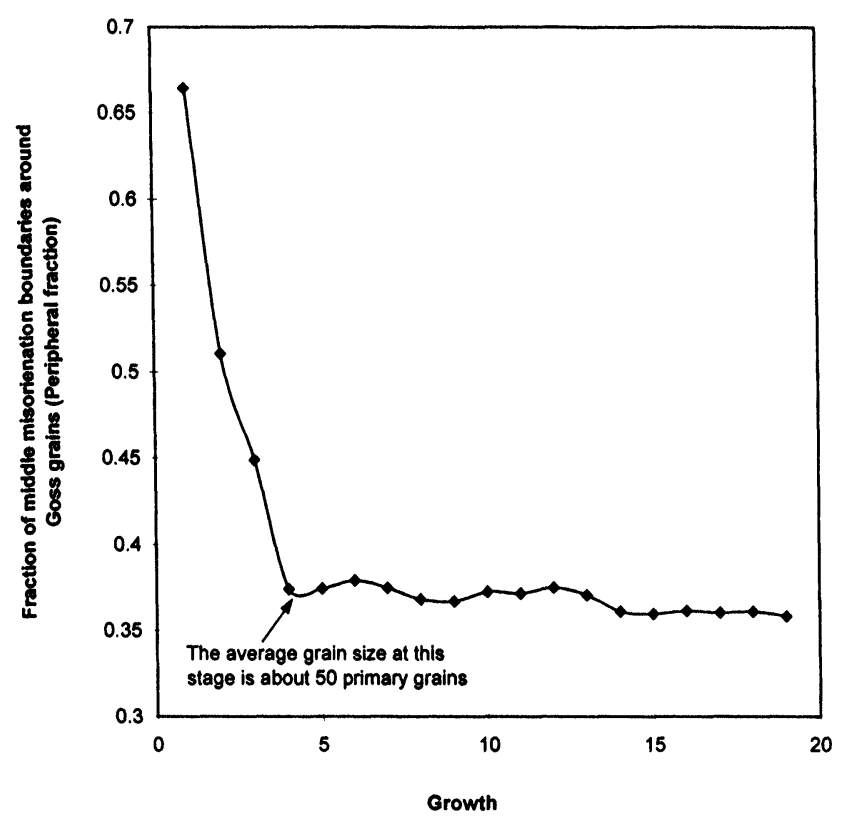

FIGURE 11 Peripheral fraction of the 'allowed' middle misorientation boundaries around the growing Goss grains as a function of growth step for the case of noninterfering neighbours of 2 in conventional $\mathrm{Fe}-3 \% \mathrm{Si}$ steel. The steady state fraction corresponds to the steady state growth rate observed in AGG. 
the steady state PF fluctuates between 3.6 and 4.0. Similar values of PF are observed for the case of $70 \%$ MF shown in Fig. 9 and the average number of non-interfering neighbours equal to two. Recently, the reduction in the number of mobile HEGB is experimentally demonstrated by Hayakawa et al. (1997) by using an EBSP measurement on high permeability steels.

In experiments, abnormally growing Goss grains reach a steady state growth rate after a certain period of time (Gol'dshteyn, 1982). This is because the growth rate at any instant is also a function of the fraction of mobile boundaries around the growing grain, the steady state growth rate corresponds to the steady state PFs simulated in the computer experiments.

As far as the CSL boundaries are concerned they do not play an important role in the AGG as the value of MF under the assumption that the CSL boundaries $\Sigma 3-\Sigma 51$ are 'allowed' is always less than $30 \%$ for both the conventional and the high permeability $\mathrm{Fe}-3 \% \mathrm{Si}$ steels. If MF is less than $30 \%$, the grain never grows abnormally (refer to Figs. 9 and 10) because the critical value required for AGG is not reached. All the presented results of the computer experiments can be used to support the conclusion that the CSL boundaries are not responsible for the $\mathrm{AGG}$ in $\mathrm{Fe}-3 \% \mathrm{Si}$ steels.

\section{CONCLUSION}

The $\mathrm{SR}$ in $\mathrm{Fe}-\mathrm{Si}$ steels is dominated by the influence of HEGBs which have a high mobility and high grain boundary diffusion coefficient. The fraction of GBs having a misorientation angle between $20^{\circ}-45^{\circ}$ is the highest around the Goss grain.

The Goss grain in the primary recrystallized $\mathrm{Fe}-\mathrm{Si}$ steel matrix has a fraction of HEGBs $\left(20^{\circ}-45^{\circ}\right.$ misorientation) that is about $\sim 40 \%$ higher than for the main texture component in both conventional and high permeability steels. On the other hand, the difference in the fraction of CSL boundaries around the Goss grain and the average texture component is only $3 \%$. Such a difference is too small to influence the abnormal growth of Gross grains.

The grain growth simulated using the Monte-Carlo method with an assumption that HEGBs have high mobility resulted in the AGG of 
Goss orientation. The assumption of high mobility of CSL boundaries does not lead to the development of Goss texture.

The assumption of assigning a high mobility to grain boundaries in the interval of $20^{\circ}-45^{\circ}$ misorientation angle satisfactorily reproduces the experimentally observed changes in the GBCD around the Goss grain during the grain growth.

The importance of the number fractions of mobile boundaries is discussed. The orientation that grows abnormally requires a high fraction of high mobility boundaries in the recrystallized matrix. In the computer experiments, a steady state peripheral fraction (PF) around the growing grains is reached during their growth. This steady state PF corresponds to the steady state growth rate generally observed at the initial stages of AGG.

\section{References}

Abbruzzese, G., Fortunati, S. and Campopiano, A. (1992). Mat. Sci. Forum, 94-96, 405. Andersen, I., Grong, O. and Ryum, N. (1995a). Acta Metall. Mater., 43(7), 2688.

Andersen, I., Grong, O. and Ryum, N. (1995b). Acta Metall. Mater., 43(7), 2689.

Anderson, M.P., Srolovitz, D.J., Grest, G.S. and Sahni, P.S. (1984). Acta Metall., 32(5), 783.

Aust, K.T. and Rutter, J.W. (1959a). Trans. Metall. Soc. AlME, 215, 119.

Aust, K.T. and Rutter, J.W. (1959b). Trans. Metall. Soc. AIME, 215, 820.

Babcock, S.E. and Balluffi, R.W. (1989). Acta Metall., 37, 2367.

Borisov, V.T., Golikov, V.M. and Scherbedinskiy, G.V. (1964). Phys. Met. Metall., $17,80$.

Bunge, H.J. (1982). In Analysis in Materials Science, Butterworths, London.

Dunn, C.G. and Lionetti, F. (1949). AIME Trans., 185, 125.

Dunn, C.G., Daniels, F.W. and Bolton, M.J. (1950a). AIME Trans., 188, 368.

Dunn, C.G., Daniels, F.W. and Bolton, M.J. (1950b). AIME Trans., 188, 1245.

Gol'dshteyn, V.Y.A., Bobkova, O.P. and Nitskaya, S.G. (1982). Phys. Met. Metall., $54(3), 85$.

Goss, N.P. (1934). Trans. ASM, 23, 511.

Gottstein, G. and Schwarzer, F. (1992). Mater. Sci. Forum, 94-96, 187.

Grimmer, H. (1974). Acta Cryst., A30, 685.

Hahn, W. and Gleiter, H. (1980). Acta Metall., 29, 601.

Harase, J. (1992). Mater. Sci. Forum, 94-96, 419.

Harase, J. and Shimizu, R. (1988). Trans. Iron Steel Inst. Jpn., 29, 388.

Harase, J., Shimizu, R., Kuroki, K., Nakayama, T., Wada, T. and Watanabe, T. (1986). Proc. 4th Japan Inst. Metals Symp. on Grain Boundary Structure and Related Phenomena, Supplement to Trans. Japan Inst. Metals, 27, 563.

Harase, J., Shimizu, R. and Dingley, D.J. (1991). Acta Metall. Mater., 39(5), 763.

Hayakawa, Y., Muraki, M. and Szpunar, J.A. (1998). Acta Mater. (to be published).

Hayakawa, Y., Szpunar, J.A., Palumbo, G. and Lin, P. (1996). Journal of Magnetism and Magnetic Materials, 160, 143.

Hayakawa, Y. and Szpunar, J.A. (1997a). Acta Mater., 45(3), 1285.

Hayakawa, Y. and Szpunar, J.A. (1997b). Acta Mater., 45(11), 4713. 
Hinz, D. and Szpunar, J.A. (1995). Phys. Rev., B52, 9900.

Humphreys, F.J. and Hatherly, M. (1995). Recrystallization and Related Annealing Phenomena, Pergamon, U.K.

Lin, P., Palumbo, G., Harase, J. and Aust, K.T. (1996). Acta Mater., 44(12), 4677.

Inokuti, Y. and Maeda, C. (1984). Trans. Iron Steel Inst. Jpn., 24, 657.

Matsuo, M. (1989). ISIJ International, 29, 809.

Morawiec, A., Szpunar, J.A. and Hinz, D.C. (1993). Acta Metall. Mater., 41(10), 825.

Pease, N.C., Jones, D.W., Wise, M.H.L. and Hutchison, W.B. (1985). Met. Sci., 15, 203.

Pelleg, J. (1966). Phil. Mag., 14, 595.

Rajmohan, N., Szpunar, J.A. and Hayakawa, Y. (1998). Acta Mater. (to be published).

Ralph, B., Shim, K.B., Huda, Z., Forley, J. and Edirisinghe, M. (1992). Mater. Sci. Forum, 94-96, 129.

Shimizu, R., Harase, J. and Dingley, D.J. (1990). Acta Metall. Mater., 38(6), 973.

Shimizu, R. and Harase, J. (1989). Acta Metall., 37, 1241.

Smith, C.S. (1952). In Metal Interfaces, 65, ASM, Cleveland.

Srolovitz, D.J., Anderson, M.P., Sahni, P.S. and Grest, G.S. (1985). Acta Metall., 33, 2233:

Titorov, D.B. (1973). Fiz. Metal. Metalloved., 35(6), 1299.

Titorov, D.B. (1994). The Physics of Metals and Metallography, 77(1), 73.

Watanabe, T. (1992). Mater. Sci. Forum, 94, 209.

Yoshitomi, Y., Ushigami, Y., Harase, J., Nakayama, T., Matsui, H. and Takahashi, N. (1993). Mater. Sci. Forum, 113-115, 715. 